\title{
Argon Physisorption as Structural Probe for Endohedrally Doped Silicon Clusters
}

\author{
Ewald Janssens, ${ }^{1,2}$ Philipp Gruene, ${ }^{3}$ Gerard Meijer, ${ }^{3}$ Ludger Wöste,${ }^{2}$ Peter Lievens, ${ }^{1, *}$ and André Fielicke ${ }^{3, \dagger}$ \\ ${ }^{1}$ Laboratory of Solid State Physics and Magnetism \& INPAC-Institute for Nanoscale Physics and Chemistry, Katholieke Universiteit \\ Leuven, Celestijnenlaan 200 D, B-3001 Leuven, Belgium \\ ${ }^{2}$ Institut für Experimentalphysik, Freie Universität Berlin, Arnimallee 14, D-14195 Berlin, Germany \\ ${ }^{3}$ Fritz-Haber-Institut der Max-Planck-Gesellschaft, Faradayweg 4-6, D-14195 Berlin, Germany
}

(Received 30 December 2006; published 9 August 2007)

\begin{abstract}
We report on an element-dependent critical size for argon physisorption at $80 \mathrm{~K}$ on transition-metaldoped silicon clusters. Argon does not attach to elemental silicon clusters but only to surface-located transition-metal atoms. Thus physisorption provides structural information. Specifically, the minimal cluster size for the formation of endohedral singly metal-doped silicon cages has been determined. The observed critical size for doubly doped silicon clusters indicates that larger caged molecules can be formed, eventually leading to the growth of metal-doped silicon nanorods.
\end{abstract}

DOI: 10.1103/PhysRevLett.99.063401

Nanometer sized parts of material exhibit nonscalable properties that strongly deviate from their bulk counterparts. Many studies investigated the nontrivial influence of the particle dimensions and the vast possibilities to tailor their electronic, optical, magnetic, and chemical properties have opened up an interdisciplinary research field [1]. Even more options arise by engineering the particle's composition [2]. For example, the optical [3] and magnetic properties of clusters triggered quests for biotechnological applications, like medical imaging, drug delivery, and nanosensors [4]. Biocompatibility requirements render many elements unsuitable for in vivo applications; commonly considered nontoxic nanoparticles include organic compounds and noble metals, as well as silicon.

The ongoing trend towards miniaturization in microelectronics has also triggered the interest in small particles made of silicon, the most used element in the semiconductor industry. Elemental silicon clusters, however, are unsuitable as building blocks for future nanomaterials, since their dangling bonds make them chemically reactive [5]. Contrary to carbon fullerenes and nanotubes, $s p^{2}$ hybridized silicon clusters are unstable and the formation of a silicon hollow cage or tube unlikely. Proper metal doping can compensate this deficiency. Recent investigations on metal-encapsulated caged silicon clusters opened up a new research avenue for novel silicon devices [6-9]. As compared to elemental silicon clusters, doped clusters exhibit higher stabilities and more symmetric structures, making them attractive for cluster assembled materials. For instance, metal-encapsulated silicon clusters are predicted to exhibit luminescence in the visible range, which is of interest for opto-electronic applications [9].

In recent years, a multitude of computational studies has been devoted to investigate the structures of transitionmetal (TM) doped silicon clusters, suggesting cagelike geometries from a certain size onwards [7-18]. It was found that incorporating a single TM atom may dramatically influence the structure and stability of the clusters. For example, doping silicon clusters with $\mathrm{Ti}$ results in
PACS numbers: 36.40.Mr, 36.40.Jn, 61.46.Bc

fullerenelike clusters and Frank-Kasper polyhedrons [11]. If, however, the number of $\mathrm{Si}$ atoms does not suffice to enclose the dopant atom completely, then basketlike geometries result $[11,13]$. It also was suggested that for multiply metal-doped silicon systems, endohedral silicon nanorods could be formed [18-20].

To date, the experimental confirmation of these predictions has been limited to indirect mass spectrometric observations $[17,21,22]$ and photoelectron spectroscopy [2325], as well as reactivity [24,25] and fragmentation studies [26]. For the multiply doped analogues there is little gas phase experimental data available. High abundances recorded for, e.g., $\mathrm{Si}_{16} \mathrm{Ti}, \mathrm{Si}_{15} \mathrm{Cr}^{+}, \mathrm{Si}_{15} \mathrm{Mn}^{+}, \mathrm{Si}_{10} \mathrm{Cu}^{+}$, $\mathrm{Si}_{15} \mathrm{~W}, \mathrm{Si}_{16} \mathrm{Mo}$, in mass spectrometric investigations are rather a sign of the enhanced stability of these sizes than a proof of their cagelike structures [21-23]. More quantitatively, photoelectron spectroscopy has confirmed the large gap between the highest occupied and the lowest unoccupied molecular orbital and high stability of $\mathrm{Si}_{16} \mathrm{Ti}$ [23]. Indications for a high stability of certain $\mathrm{Si}_{n} X^{+}(n=9-14)$ clusters have been obtained by Kanayama et al. who reported on the reaction of TM atoms (Hf, Ta, W, Re, and $\mathrm{Ir}$ ) with silane $\left(\mathrm{SiH}_{4}\right)$ [6]. That experiment resulted in magic cluster sizes characterized by weak bonding of hydrogen, which might be explained by the formation of endohedral structures. Recently, Ohara et al. studied the size-dependent reactivity towards $\mathrm{H}_{2} \mathrm{O}$ vapor in a flow tube reactor [24,25]. The abundance of $\mathrm{Si}_{n} \mathrm{Ti}^{+}(n=7-11)$ decreased upon reaction with $\mathrm{H}_{2} \mathrm{O}$, while the abundance of larger $\mathrm{Si}_{n} \mathrm{Ti}^{+}(n=13-17)$ clusters remained unchanged. An exterior Ti atom is a reactive site for adsorption of $\mathrm{H}_{2} \mathrm{O}$. The low reactivity of larger $\mathrm{Si}_{n} \mathrm{Ti}^{+}$clusters indicates that these clusters have no exterior $\mathrm{Ti}$ atom [24,25].

For metal clusters, Knickelbein and Menezes have proposed that the size-dependent efficiency of rare gas physisorption reflects changes in the cluster surface topology [27]. Ar is an ideal probe: it is expected to have a negligible influence on the cluster and merely serves as a spectator atom [27-29]. Ar has no internal degrees of freedom and 
represents a good compromise in terms of polarizability: it can be attached to the cluster ion without much perturbation to the electronic structure.

In this Letter we demonstrate that the physisorption of Ar acts as a structural probe for TM doped silicon clusters and is very sensitive to the position of the TM atom(s) in the cluster. In particular, we provide experimental evidence for the onset of the formation of endohedral structures as a function of cluster size and dopant element.

The binary $\mathrm{Si}_{n} X_{m}{ }^{+}$clusters are produced in a $10 \mathrm{~Hz}$ dual-target dual-laser vaporization source [30,31]. Two independent $\mathrm{Nd}$ : YAG lasers vaporize the target materials and create a plasma. Subsequent injection of a short pulse of helium cools the plasma and leads to condensation in the clustering channel. This condensation room is followed by a thermalization room, a $30 \mathrm{~mm}$ long channel drilled in a $\mathrm{Cu}$ block, which is thermally isolated from the main body of the source and cooled by a continuous flux of liquid nitrogen. A temperature controller allows for stabilization to any temperature in the $80-320 \mathrm{~K}$ range. If a fraction (typically 1\%) of Ar is added to the He carrier gas, clusterargon complexes can be formed. After expansion into vacuum the cluster distribution in the molecular beam is analyzed using a reflectron time-of-flight mass spectrometer $[29,32]$.

Figure 1 shows mass spectra obtained for mixed $\mathrm{Si}_{n} X_{m}{ }^{+}$ $(X=\mathrm{V}$ and $\mathrm{Cr}$ ) clusters after adding $1 \%$ of Ar to the $\mathrm{He}$

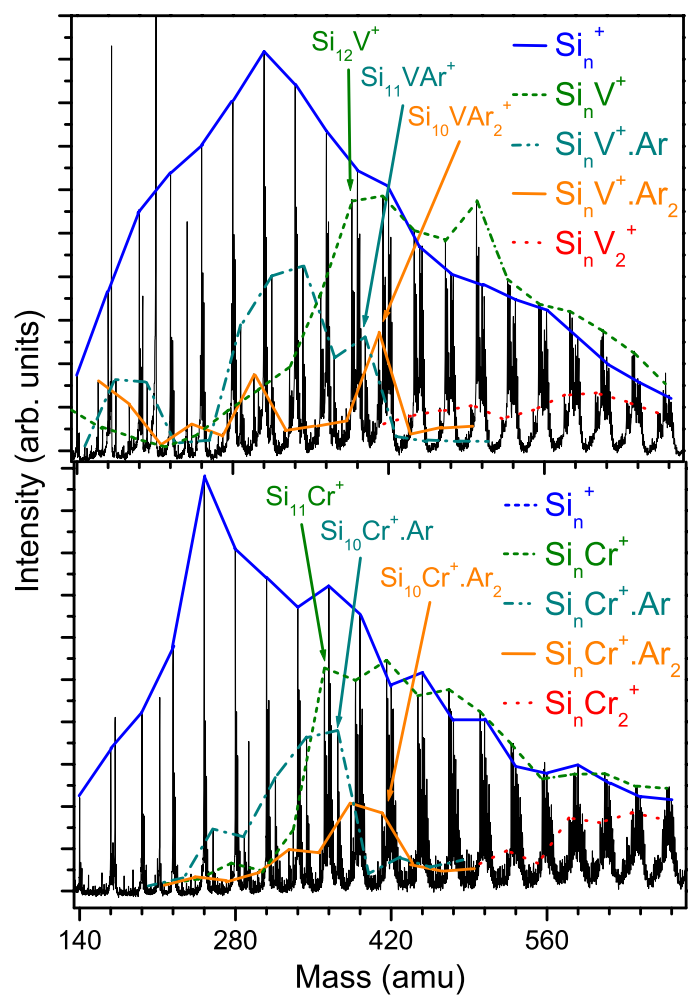

FIG. 1 (color online). Mass spectra of $\operatorname{Si}_{n} X_{m}^{+}(X=\mathrm{V}, \mathrm{Cr})$ clusters formed at $80 \mathrm{~K}$ using $\mathrm{He}$ carrier gas containing $1 \%$ Ar. $\mathrm{Si}_{n}^{+}, \mathrm{Si}_{n} X_{1,2}^{+}$, and $\mathrm{Si}_{n} X^{+} \cdot \mathrm{Ar}_{1,2}$ peak maxima are connected by solid lines. carrier gas. The mass spectra are congested due to the large number of possible compositions and due to the natural isotope distributions of the elements used. The highest ion signals are recorded for bare $\mathrm{Si}_{n}^{+}$and singly doped $\mathrm{Si}_{n} X^{+}$. There is also a fraction of $\mathrm{Si}_{n} X_{2}^{+}$clusters and $\mathrm{Si}_{n} X_{2}{ }^{+} \cdot \mathrm{Ar}_{1,2}$ complexes, but for bare $\mathrm{Si}_{n}^{+}$no argon complexes are formed. We note here that we have observed rare gas complex formation under similar conditions using larger and more polarizable $\mathrm{Xe}$ atoms, as it has been reported before by Antonietti et al. [33].

Most remarkably, the abundance of the $\mathrm{Si}_{n} X_{1,2}^{+} \cdot \mathrm{Ar}_{1,2}$ complexes is strongly size dependent and collapses after a certain critical number of $\mathrm{Si}$ atoms. Beyond this critical size the intensity of the corresponding $\mathrm{Ar}$-free $\mathrm{Si}_{n} X_{1,2}^{+}$ clusters abruptly increases. Similar effects are observed for the other investigated systems $\left(\mathrm{Si}_{n} X_{1,2}^{+}, X=\mathrm{Ti}\right.$, Co, $\mathrm{Cu}$ ), which is best represented by plotting the fraction of $\mathrm{Ar}$ complexes (one or two Ar atoms) as function of $n$ (see Fig. 2). The abundance of each cluster has been obtained by fitting the expected isotope distribution to the measured mass spectra. The error bars give the standard deviation on the average of several independently taken spectra and account for the uncertainty introduced by the fitting procedure. An extended description and additional figures can be found in the supplementary information [34]. Both for singly and doubly doped silicon clusters critical sizes for argon attachment are observed, which depend on the dopant element. For singly doped silicon clusters the critical size for Ar attachment changes along the $3 d$ row, i.e., $\mathrm{Si}_{12} \mathrm{Ti}^{+}, \mathrm{Si}_{11} \mathrm{~V}^{+}, \mathrm{Si}_{10} \mathrm{Cr}^{+}, \mathrm{Si}_{7} \mathrm{Co}^{+}, \mathrm{Si}_{11} \mathrm{Cu}^{+}$. Knowing that $\mathrm{Si}_{n}$ clusters do not form stable complexes with $\mathrm{Ar}$ at $80 \mathrm{~K}$, it is reasonable to assume that Ar binds to the TM dopant. This assumption is supported by the observed magnitude of the Ar binding energy to the cluster (see below). Binding to the TM atom is only feasible if the dopant is on the surface of the host cluster (exohedral). If the dopant resides in the interior of a $\mathrm{Si}_{n}$ cage (endohedral), the Ar atom can only interact with Si surface atoms, thus no $\mathrm{Ar}$ complexes are formed. Since less $\mathrm{Si}$ atoms are needed to fully cover a smaller dopant, the experiments are in good agreement with the decreasing atomic radius along the $3 d$ row and the atomic radii of $\mathrm{Cu}$ and $\mathrm{V}$ being similar. Thus, Ar attachment is a probe for an uncompleted caged structure: the disappearance of the Ar complexes marks the formation of endohedral clusters.

To get a quantitative understanding of the strength of the Ar-cluster bond and its scaling with cluster size, we have studied the temperature dependence of the complex formation by equilibrium concentration measurements, one of the most accurate ways to determine relative Gibbs energies. This methodology has been used before to obtain binding energies of neutral ligands to metal clusters [35]. The Ar physisorption is investigated in the $80-300 \mathrm{~K}$ temperature range for $\mathrm{Si}_{n} \mathrm{~V}^{+}(n=7-11)$ :

$$
\mathrm{Si}_{n} \mathrm{~V}^{+}+\mathrm{Ar} \rightleftharpoons \mathrm{Si}_{n} \mathrm{~V}^{+} \cdot \mathrm{Ar}
$$




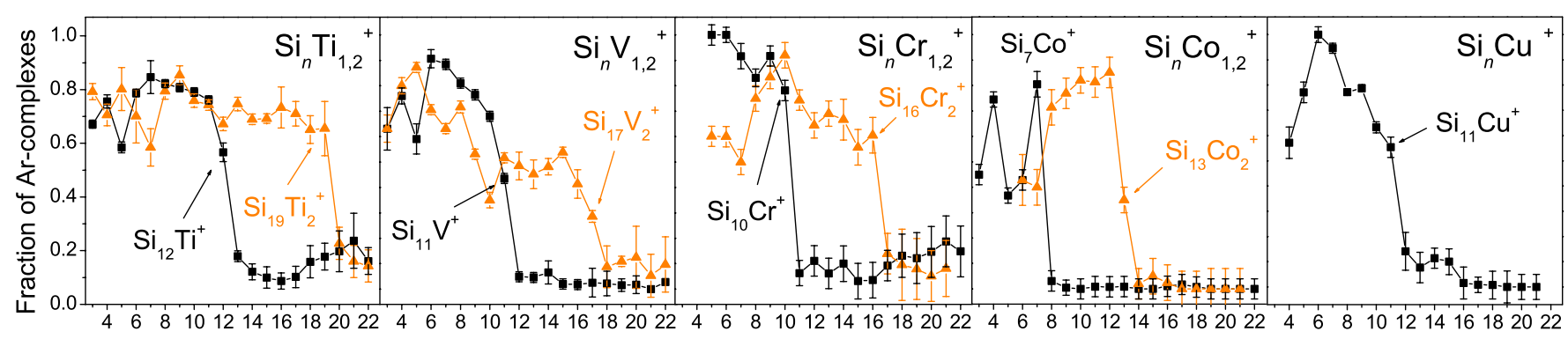

$n$

FIG. 2 (color online). Fraction of argon complexes formed for $\mathrm{Si}_{n} X^{+}(X=\mathrm{Ti}, \mathrm{V}, \mathrm{Cr}, \mathrm{Co}, \mathrm{Cu})$ as a function of cluster size. A critical size, beyond which the argon-complex formation is unlikely, is found for both the singly and doubly doped species.

The recorded intensities $I\left(\mathrm{Si}_{n} \mathrm{~V}^{+} \cdot \mathrm{Ar}\right)$ and $I\left(\mathrm{Si}_{n} \mathrm{~V}^{+}\right)$reflect equilibrium intensities, as long as there is an equilibrium in the thermalization room, which is frozen out afterwards. The cluster argon complexes may neither dissociate after leaving the source nor be formed during the expansion. Although the number of collisions is limited during the expansion, it is unlikely that these conditions are fully satisfied. The equilibrium constant $K^{0}$ with the standard pressure $p^{0}$ is given by:

$$
K^{0}=\frac{I\left(\mathrm{Si}_{n} \mathrm{~V}^{+} \cdot \mathrm{Ar}\right)}{I\left(\mathrm{Si}_{n} \mathrm{~V}^{+}\right) p(\mathrm{Ar}) / p^{0}}
$$

The total pressure in the thermalization channel cannot be measured directly, but is roughly estimated to be $30 \mathrm{mbar}$ at room temperature. The gas is supposed to behave ideally and the Ar partial pressure $p(\mathrm{Ar})$ decreases linearly with decreasing temperature. According to the GibbsHelmholtz equation the equilibrium constant $K^{0}$ is related to the standard enthalpy change $\Delta H^{0}$ and the standard entropy change $\Delta S^{0}$ :

$$
\ln K^{0}=\frac{-\Delta H^{0}}{R} \frac{1}{T}+\frac{\Delta S^{0}}{R} .
$$

Exact determination of $\Delta S^{0}$ is impossible, because of the uncertainty of $p(\mathrm{Ar})$. The slope of a plot of $\ln K^{0}$ versus the reciprocal temperature (van't Hoff plot) yields $-\Delta H^{0}$, which equals the cluster-argon bond dissociation energy (BDE) under constant pressure conditions. Because of the above-mentioned limitations, the slope of the linear part in the van't Hoff plots (see Fig. 3) does not allow for a precise measurement of the BDE, but rather for an estimation of the order of magnitude. The linear regression of the curves results in errors of about $0.01 \mathrm{eV}$. The obtained values are given in Fig. 3, and are in the $0.12-0.16 \mathrm{eV}$ range. The magnitude of these values is consistent with the calculated binding energy for argon on $\mathrm{Au}_{4}^{+}$of $0.12 \mathrm{eV}$ [36], and with typical adsorption energies of rare gases on metal surfaces $(0.1-0.2 \mathrm{eV})$ [37]. A size-dependent trend can be detected; $\triangle H^{0}$ decreases with increasing cluster size. This can be explained by the better accessibility of a less encapsulated $\mathrm{V}$ dopant. Additionally, the positive charge is more smeared out in larger clusters, yielding a smaller BDE.
It has been predicted computationally that the minimal cage size depends on the radius of the dopant atom and the strength of the $\mathrm{Si}-X$ interactions $[9,14]$. Most computations are performed on neutral clusters, while the experiments are done on cations. Nevertheless, several studies have shown that the charge state has a minor influence on the geometry of $\mathrm{Si}_{n} X$ clusters $[10,12,13]$. It was predicted by Khanna et al. that $\mathrm{Cr}$ is small enough to occupy the center of $\operatorname{Si}_{n}(n=11-14)$ cages [8]. Kumar and Kawazoe, however, found caged structures for $\mathrm{Si}_{n} \mathrm{Cr}$ from $n=12$ onwards and open basketlike structures for $n<12$ [12]. Our experiments identify $\mathrm{Si}_{11} \mathrm{Cr}^{+}$as the smallest endohedral $\mathrm{Si}_{n} \mathrm{Cr}^{+}$cluster. The proposed basket shaped structures for $\mathrm{Si}_{n} \mathrm{Ti}(n=8-12)$ and endohedral systems for larger sizes [13] agree nicely with the experiment. However, the exohedral structure of $\mathrm{Si}_{9} \mathrm{Co}$ found in Ref. [16] disagrees with the disappearance of the Ar complexes from $\mathrm{Si}_{8} \mathrm{Co}$ onwards. Hagelsberg et al. have shown that $\mathrm{Si}_{12} \mathrm{Cu}$ stabilizes in a cage geometry, while in $\mathrm{Si}_{10} \mathrm{Cu}$ the $\mathrm{Cu}$ atom occupies a surface site [10]. Again, the present experiments support the computations. Moreover, earlier experimental work also supports our findings. Ohara et al. showed that the reaction probability of $\mathrm{Si}_{n} \mathrm{Ti}^{+}$towards $\mathrm{H}_{2} \mathrm{O}$ drops off in the $n=11-13$ range [24]. Jaeger et al. investigated the fragmentation behavior of selected $\mathrm{Si}_{n} X^{+}$clusters, and came to the conclusion that $\mathrm{Cr}$ and $\mathrm{Cu}$ are weakly bound

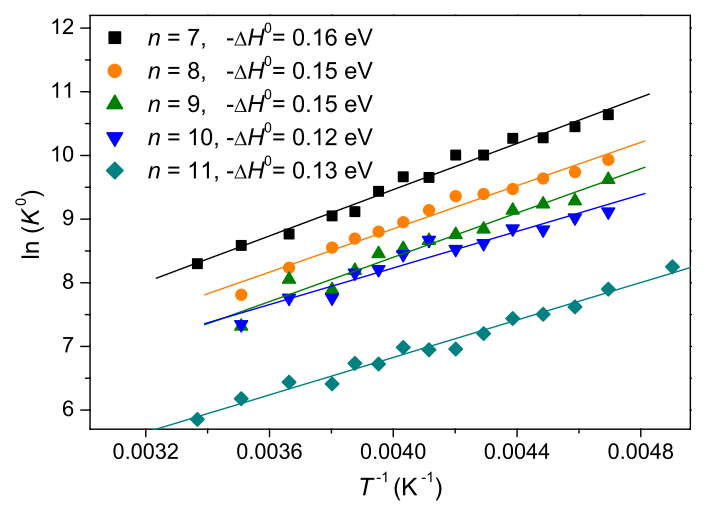

FIG. 3 (color online). Linear part of the van't Hoff plots for $\mathrm{Si}_{n} \mathrm{~V}^{+}(n=7-11) . \Delta H^{0}$ values have been extracted from the slope of the fits. 
surface atoms in $\mathrm{Si}_{7} \mathrm{Cu}^{+}, \mathrm{Si}_{10} \mathrm{Cu}^{+}$, and $\mathrm{Si}_{7} \mathrm{Cr}^{+}$, while $\mathrm{Cr}$ is strongly bound in $\mathrm{Si}_{15} \mathrm{Cr}^{+}$[26].

Additionally, for doubly doped silicon clusters, the critical size for Ar attachment decreases along the $3 d$ row: $\mathrm{Si}_{19} \mathrm{Ti}_{2}^{+}, \mathrm{Si}_{17} \mathrm{~V}_{2}^{+}, \mathrm{Si}_{16} \mathrm{Cr}_{2}^{+}, \mathrm{Si}_{13} \mathrm{Co}_{2}^{+}$. It is again reasonable to assume that no Ar-complex formation is possible if the dopant atoms are fully surrounded by $\mathrm{Si}$. The gradual transition of the Ar sticking probability for $\mathrm{Si}_{n} \mathrm{~V}_{2}^{+}$and $\mathrm{Si}_{n} \mathrm{Co}_{2}^{+}$could be due to the coexistence of endohedral and exohedral isomers in this size range. Using only the experimental data, one intriguing question is left unanswered: do the dopant atoms form a dimer inside a big cage or do several singly doped cages link together?

Only a few theoretical studies are available on this topic. Pioneering work by Andriotis et al. and Singh et al. predict the existence of one-dimensional chains of metal atoms that can stabilize silicon nanorods which encapsulate the metal atom chain [18-20]. To date, however, there is no experimental confirmation. Formation of TM encapsulated $\mathrm{Si}_{6 n+6} \mathrm{~V}_{n}$ and $\mathrm{Si}_{5 n+7} \mathrm{Ni}_{n}$ silicon nanorods could be modeled by adding $\mathrm{Si}_{6} \mathrm{~V}$ and $\mathrm{Si}_{5} \mathrm{Ni}$ units to endohedral $\mathrm{Si}_{12} \mathrm{~V}$ or $\mathrm{Si}_{12} \mathrm{Ni}$ clusters $[18,19]$. The predicted sizes are in perfect agreement with the observed disappearance of the argoncomplex formation at $\mathrm{Si}_{12} \mathrm{~V}^{+}$and $\mathrm{Si}_{18} \mathrm{~V}_{2}^{+}$. In $\mathrm{Si}_{18} \mathrm{~V}_{2}$, the $\mathrm{V}$ atoms are only $1.97 \AA$ separated and their mutual interaction cannot be neglected [18]. Kumar and Kawazoe also predict $\mathrm{Si}_{6 n+6} \mathrm{Cr}_{n}$ nanorods [9]. Their TM encapsulated double prism structure for $\mathrm{Si}_{18} \mathrm{Cr}_{2}$ is in agreement with the present experiment. $\mathrm{Si}_{n} X_{2}$ clusters can thus consist of two strongly interconnected cages and could be seen as the onset to the formation of silicon nanorods with enclosed metal atoms.

In summary, we have shown that structural properties of silicon clusters doped with TM atoms can be probed by exploiting their ability to physisorb Ar atoms. Ar does not attach to elemental silicon clusters, but only to the TM doped clusters if the number of $\mathrm{Si}$ atoms is smaller than a certain critical size. The critical size for Ar attachment depends on the size of the TM dopant and is linked to the formation of caged clusters, in good agreement with earlier computational predictions. Also for doubly doped $\mathrm{Si}_{n} X_{2}^{+}$ clusters, a critical size for Ar attachment is found, which might indicate that larger endohedral caged molecules are formed, eventually leading to the growth of metal-doped silicon nanorods.

This work is supported by the Fund for Scientific Research-Flanders (FWO), the Flemish Concerted Action (No. GOA/2004/02), and the Belgian Interuniversity Poles of Attraction (No. IAP/P5/01) programs. E. J. received additional support from the FWO. Support by the FOM Institute for Plasmaphysics in Nieuwegein (NL) is gratefully acknowledged.

*peter.lievens@fys.kuleuven.be †fielicke@fhi-berlin.mpg.de
[1] Atomic Clusters: From Gas Phase to Deposited, edited by D. Woodruff, The Chemical Physics of Solid Surfaces Vol. 12 (Elsevier, New York, 2007).

[2] E. Janssens et al., Phys. Rev. Lett. 96, 233401 (2006).

[3] L. A. Peyser et al., Science 291, 103 (2001).

[4] G. M. Whitesides, Nat. Biotechnol. 21, 1161 (2003).

[5] U. Röthlisberger, W. Andreoni, and M. Parrinello, Phys. Rev. Lett. 72, 665 (1994).

[6] H. Hiura, T. Miyazaki, and T. Kanayama, Phys. Rev. Lett. 86, 1733 (2001).

[7] V. Kumar, Comput. Mater. Sci. 36, 1 (2006).

[8] S. N. Khanna, B. K. Rao, and P. Jena, Phys. Rev. Lett. 89, 016803 (2002).

[9] V. Kumar and Y. Kawazoe, Phys. Rev. Lett. 90, 055502 (2003).

[10] F. Hagelberg, C. Xiao, and W. A. Lester, Jr., Phys. Rev. B 67, 035426 (2003).

[11] V. Kumar, T. M. Briere, and Y. Kawazoe, Phys. Rev. B 68, 155412 (2003).

[12] H. Kawamura, V. Kumar, and Y. Kawazoe, Phys. Rev. B 70, 245433 (2004)

[13] H. Kawamura, V. Kumar, and Y. Kawazoe, Phys. Rev. B 71, 075423 (2005).

[14] C. Majumder and S. K. Kulshreshtha, Phys. Rev. B 70, 245426 (2004).

[15] G. K. Gueorguiev et al., Thin Solid Films 515, 1192 (2006).

[16] J. Lu and S. Nagase, Phys. Rev. Lett. 90, 115506 (2003).

[17] Z. F. Chen et al., J. Am. Chem. Soc. 128, 12829 (2006).

[18] A. N. Andriotis et al., New J. Phys. 4, 78 (2002).

[19] M. Menon, A. N. Andriotis, and G. E. Froudakis, Nano Lett. 2, 301 (2002).

[20] A. K. Singh et al., Nano Lett. 2, 1243 (2002).

[21] S. M. Beck, J. Chem. Phys. 87, 4233 (1987).

[22] S. Neukermans et al., Int. J. Mass Spectrom. 252, 145 (2006).

[23] K. Koyasu et al., J. Am. Chem. Soc. 127, 4998 (2005).

[24] M. Ohara et al., Chem. Phys. Lett. 371, 490 (2003).

[25] M. Ohara et al., J. Phys. Chem. A 106, 3702 (2002).

[26] J. B. Jaeger, T.D. Jaeger, and M. A. Duncan, J. Phys. Chem. A 110, 9310 (2006).

[27] M. B. Knickelbein and W. J. C. Menezes, J. Phys. Chem. 96, 6611 (1992).

[28] A. Fielicke et al., Phys. Rev. Lett. 93, 023401 (2004).

[29] A. Fielicke, G. von Helden, and G. Meijer, Eur. Phys. J. D 34, 83 (2005).

[30] W. Bouwen et al., Rev. Sci. Instrum. 71, 54 (2000).

[31] E. Janssens et al., Phys. Rev. B 69, 085402 (2004).

[32] G. von Helden, D. van Heijnsbergen, and G. Meijer, J. Phys. Chem. A 107, 1671 (2003).

[33] J. M. Antonietti et al., Phys. Rev. B 68, 035420 (2003).

[34] See EPAPS Document No. E-PRLTAO-99-048733 for $\mathrm{Si}_{n} X_{m}{ }^{+}$mass spectra and for the cluster intensities taking into account isotopomeric distributions. For more information on EPAPS, see http://www.aip.org/pubservs/ epaps.html.

[35] E. K. Parks et al., J. Chem. Phys. 108, 3731 (1998).

[36] A. Schweizer et al., J. Chem. Phys. 119, 3699 (2003).

[37] L.W. Bruch, M.W. Cole, and E. Zaremba, Physical Adsorption: Forces and Phenomena (Clarendon, Oxford, 1997), p. 229. 